\title{
The difference in expression of long noncoding RNAs in rat semen induced by high-fat diet was associated with metabolic pathways
}

\author{
Tian An ${ }^{1}$, Hui Fan ${ }^{1}$, Yu F Liu ${ }^{2}$, Yan Y Pan ${ }^{1}$, Ying K Liu ${ }^{3}$, Fang F Mo ${ }^{1}$, Yu J Gu ${ }^{1}$, Ya L Sun ${ }^{4}$, Dan D Zhao \\ ${ }^{1}$, Na Yu ${ }^{1}$, Yue Ma ${ }^{1}$, Chen Y Liu ${ }^{1}$, Qiu L Wang ${ }^{3}$, Zheng Y Li ${ }^{1}$, Fei Teng ${ }^{1,5}$, Si Hua Gao ${ }^{\text {Corresp.. }}{ }^{1}$, Guang J \\ Jiang Corresp. 1 \\ ${ }^{1}$ Diabetes Research Center, Beijing University of Chinese Medicine, Beijing, China \\ 2 Beijing University of Chinese Medicine Third Affiliated Hosiptal, Beijing, China \\ 3 Beijing He ping li Hospital, Beijing, China \\ 4 Beijing Chang ping Chinese Medicine Hospital, Beijing, China \\ 5 State Key Laboratory of Stem Cell and Reproductive Biology, Institute of Zoology, Chinese Academy of Sciences, Beijing, 100101, China., Beijing, China \\ Corresponding Authors: Si Hua Gao, Guang J Jiang \\ Email address: sihuagaopaper@sina.com, guangjianjiang@sina.com
}

Background. Obesity, a common metabolic disease, is a known cause of male infertility due to its associated health risk. Long noncoding RNAs (IncRNAs) has also been reported to be associated with male reproductive diseases; however, their role in the association between high-fat diet-induced obesity (DIO) and male reproduction remains unclear.

Methods. We used microarray analysis to compare the expression levels of IncRNAs and mRNAs in the spermatozoa of rats with DIO and normal rats. We selected a few IncRNAs that were obviously up-regulated or down-regulated, and then used RT-PCR to verify the accuracy of their expression. We then performed a functional enrichment analysis of the differentially expressed mRNAs using gene ontology and pathway analysis. Finally, target gene predictive analysis was used to explore the relationship between IncRNAs and mRNAs. Results. The results revealed a statistically significant difference in the fasting blood glucose level in rats with DIO and control rats. We found that 973 IncRNAs and 2,994 mRNAs were differentially expressed in the sperm samples of the DIO rats, compared to the controls. GO enrichment analysis revealed 263 biological process terms, 39 cellular component terms, and 40 molecular function terms $(p<0.01)$ in the differentially expressed mRNAs. The pathway analysis showed that metabolic pathways were most enriched in protein-coding genes. Discussion. To the best of our knowledge, this is the first report to show differences in the expression levels of IncRNAs and mRNAs in the sperms of rats with DIO and normal rats, and to determine the expression profile of IncRNAs in the sperm of rats with DIO. Our results have revealed a number of IncRNAs and pathways associated with obesity-induced infertility, including metabolic pathways. These pathways could be new candidates that help cope with and investigate the mechanisms 
behind the progression of obesity-induced male infertility. 
1 The difference in expression of long noncoding RNAs in rat semen induced by

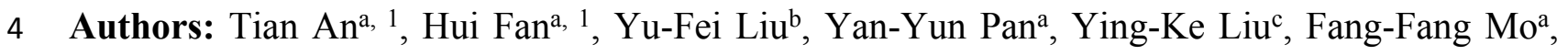
5 Yu-Jie Gua , Ya-Ling Sund, Dan-Dan Zhao a ${ }^{\mathrm{a} a ~ Y u^{\mathrm{a}}}$, Yue Ma ${ }^{\mathrm{a}}$, Chen-Yue Liu ${ }^{\mathrm{a}}$, Qiu-Li Wange, 6 Zheng-Yun Li ${ }^{\mathrm{a}}$, Fei Tenga,e, Si-Huan Gao ${ }^{\mathrm{a}^{*}}$, Guang-Jian Jiang ${ }^{\mathrm{a}^{*}}$

\section{Affiliations:}

9 a Diabetes Research Center, Beijing University of Chinese Medicine, Beijing, 100029, China

10 b Beijing University of Chinese Medicine Third Affiliated Hospital, Beijing, 100029, China

$11{ }^{\mathrm{c}}$ Beijing He ping li Hospital, Beijing, 100013, China

${ }^{d}$ Beijing Chang ping Chinese Medicine Hospital, Beijing, 102200, China

e State Key Laboratory of Stem Cell and Reproductive Biology, Institute of Zoology, Chinese Academy of Sciences, Beijing, 100101, China.

* Corresponding authors:

G.J. Jiang; E-mail: guangjianjiang@sina.com; Tel: +86-10-64286915; Fax: +86-10-64286672

S.H. Gao; E-mail: sihuagaopaper@sina.com; Tel: +86-10-64286915; Fax: +86-10-64286672

${ }^{1}$ These authors contributed equally to this work. 

48

\section{Abstract:}

Background. Obesity, a common metabolic disease, is a known cause of male infertility due to its associated health risk. Long noncoding RNAs (lncRNAs) has also been reported to be associated with male reproductive diseases; however, their role in the association between highfat diet-induced obesity (DIO) and male reproduction remains unclear.

Methods. We used microarray analysis to compare the expression levels of lncRNAs and mRNAs in the spermatozoa of rats with DIO and normal rats. We selected a few lncRNAs that were obviously up-regulated or down-regulated, and then used RT-PCR to verify the accuracy of their expression. We then performed a functional enrichment analysis of the differentially expressed mRNAs using gene ontology and pathway analysis. Finally, target gene predictive analysis was used to explore the relationship between lncRNAs and mRNAs.

Results. The results revealed a statistically significant difference in the fasting blood glucose level in rats with DIO and control rats. We found that 973 lncRNAs and 2,994 mRNAs were differentially expressed in the sperm samples of the DIO rats, compared to the controls. GO enrichment analysis revealed 263 biological process terms, 39 cellular component terms, and 40 molecular function terms $(p<0.01)$ in the differentially expressed mRNAs. The pathway analysis showed that metabolic pathways were most enriched in protein-coding genes.

Discussion. To the best of our knowledge, this is the first report to show differences in the expression levels of lncRNAs and mRNAs in the sperms of rats with DIO and normal rats, and to determine the expression profile of lncRNAs in the sperm of rats with DIO. Our results have revealed a number of lncRNAs and pathways associated with obesity-induced infertility, including metabolic pathways. These pathways could be new candidates that help cope with and investigate the mechanisms behind the progression of obesity-induced male infertility.

Keywords: Long noncoding RNA; Microarray analysis; Obesity; Sperm; Male infertility 
50

51

52

53

54

55

56

\section{Introduction:}

1 Obesity is a serious, chronic metabolic disease with several comorbidities, including non-insulindependent diabetes mellitus, high cholesterol, heart disease, hypertension, cancer, and psychological depression ${ }^{[1,2]}$. The incidence of obesity has increased rapidly in recent years, and this increase in obesity has been accompanied by a decrease in male fertility and fecundity ${ }^{[3]}$. Obesity is known to cause serious harm to the male reproductive system, in both humans and animals ${ }^{[4]}$. Studies have shown that obesity reduces spermatogenesis and fertility by affecting the volume, concentration, and motility of sperms. It also causes erectile dysfunction and variations in testicular tissue and sperm proteomics ${ }^{[5-9]}$.

Long noncoding RNAs (lncRNAs) are a group of RNAs with mRNA-like transcripts, length ranging from $200 \mathrm{nt}$ to $100 \mathrm{~kb}$, and limited protein-coding potential ${ }^{[10,11]}$. Recent studies ${ }^{[12]}$ have shown that lncRNAs can be translated to encode functional peptide segments. They can participate in various biological processes, including cell cycle regulation, differentiation, and epigenetic regulation ${ }^{[13,14]}$. Many studies, including ours, have established an association between lncRNAs and male reproduction by revealing differences in the expression profiles of lncRNAs in the spermatozoon of mice with high-fat diet-induced obesity (DIO), diabetic mice, and normal mice ${ }^{[15]}$. In a previous study, we had investigated the expression profile of lncRNAs in the sperm of diabetic mice; we found that 7721 lncRNAs and 6097 mRNAs were differentially expressed in the sperms of diabetic and normal mice ${ }^{[16]}$. Therefore, we hypothesized that the effects of obesity, a risk factor for diabetes, on spermatozoa were associated with lncRNA and its target genes.

In the current study, we aimed to explore the link between obesity and male infertility, based on lncRNA expression profile. We established a rat model of obesity induced by a high-fat diet, in order to understand how it might affect the male reproductive system at the epigenetic (lncRNA) level and to explore the possible biological processes and pathways associated with obesity and associated fertility disorders.

\section{Materials and Methods}




\subsection{Animal models and sperm collection}

All protocols in this study were approved by the Animal Care Committee of the Institute of Zoology, Chinese Academy of Sciences, and all animals were treated according to the guidelines of the Animal Care Committee. Male SD rats (6-week old; Hua Fu Kang Company, Beijing, China) were used in this study. The field experiments were approved by the Research Council of Chinese Academy of Sciences (certification number SCXK [Jing] 2011-0024). After 1 week of acclimation, the rats were randomly divided into DIO and normal groups. The rats in the DIO group were fed on a high-fat diet (20\% sucrose, $10 \%$ lard, $2.5 \%$ cholesterol, $0.2 \%$ sodium cholic acid, and 67.3\% (w/w) standard chow) for 12 weeks to induce obesity (average body weight: DIO $>(1+20 \%)$ normal, $n=7)$. The rats in the normal group $(n=7)$ were given the standard diet. Sperm was collected from the epididymis of all the rats. Sperm collection was performed according to the method described by Jiang ${ }^{[16]}$. Briefly, the rats were sacrificed by cervical dislocation; the spermatozoa were extracted and placed in preheated human tubal cultures, which were then centrifuged $\left(1500 \times \mathrm{g}, 4^{\circ} \mathrm{C}, 8 \mathrm{~min}\right)$ to collect the supernatant fluid.

\subsection{Sperm analysis and testis histomorphology evaluation}

Semen was obtained from the tail of the epididymis and quickly placed in a Centrifuge tube with $1 \mathrm{ml}$ of Ham's F10 medium (Bioway Biotechnology Co., Ltd., Beijing, China) for analysis of sperm motility and density using computer - assisted semen analyzer (CASA, Hamilton Thorne TOX IVOS, USA). Testis tissue were collected from 19-week-old DIO and normal rats, and first fixed in $4 \%$ neutral formaldehyde fixative, then embded in paraffin. Sections of $4 \mu \mathrm{m}$ thicknesses were used for hematoxylin and eosin (HE) staining, which was conducted according to convention methods. Finally, the morphological changes in testis tissue section was observed using an optical microscope (Olympus, Tokyo, Japan).

\subsection{RNA extraction}

The supernatant fluid samples from three rats each from the DIO and normal groups were selected. We extracted and purified total RNA from these samples using the miRNeasy Mini Kit (QIAGEN, GmBH, Germany) according to the manufacturer's instructions and calculated the 
104

105

106

107

108

109

110

111

112

113

114

115

116

117

118

119

120

121

122

123

124

125

126

127

128

129

130

RIN number to assess the integration of RNA using an Agilent Bioanalyzer 2100 (Agilent technologies, Santa Clara, CA, US).

\subsection{LncRNA microarray experiments}

We performed the microarray analysis using the Rat Genome Oligo nucleotide $4644 \mathrm{k}$ Microarrays (Agilent, CA, USA) at Biotechnology Corporation (SBC, China). We amplified and labeled the total RNA using the Low Input Quick Amp WT Labeling Kit (Cat.\# 5190-2943, Agilent technologies, Santa Clara, CA, US), according to the manufacturer's instructions; the labeled cRNAs were purified using the RNeasy mini kit (Cat.\# 74106, QIAGEN, GmBH, Germany). Based on the instructions in the Agilent microarray supporting kit for the Hybridization Oven (Cat.\# G2545A, Agilent technologies, Santa Clara, CA, US), the conditions for hybridization were set as $65^{\circ} \mathrm{C}$ at $10 \mathrm{rpm}$ for $17 \mathrm{~h}$, and the volume of the cRNA sample for hybridization was $1.65 \mu \mathrm{g}$. The slides were then washed in staining dishes (Cat.\# 121, Thermo Shandon, Waltham, MA, US) using the Gene Expression Wash Buffer Kit (Cat.\# 5188-5327, Agilent technologies, Santa Clara, CA, US), according to the manufacturer's instructions. The information obtained from the scanner was loaded into the image analysis program, Feature Extraction software 10.7 (Agilent technologies, Santa Clara, CA, US), and the data were normalized using the Quantile algorithm from GeneSpring Software 12.6.1 (Agilent technologies, Santa Clara, CA, US).

\subsection{Bioinformatics data analysis}

After the original data was normalized using the GeneSpring Software (Agilent technologies, Santa Clara, CA, US), we screened high-quality probes for further data analysis. We analyzed fold-change (fold-differences in expression) and used $t$-tests (Student's $t$-test) for investigating the differentially expressed genes. After the raw data from the microarray was standardized and converted to $\log 2$ values, a scatter plot was generated in a two-dimensional coordinate system. Using the online analysis software DAVID (https://david.ncifcrf.gov/), we analyzed the Gene Ontology (GO) enrichment of the differentially expressed mRNAs and their functions, based on three aspects: biological processes (BP), cellular components (CC), and molecular functions 
131 (MF). The $\log 10$ values (p-value) denote enrichment scores and represent the significance of the 132 GO term enrichment among the differentially expressed genes (DEGs). We also performed 133 KEGG pathway analysis to reveal pathway clusters covering the DEGs; here, the $\log 10$ values 134 (p-value) denote the enrichment score and represent the significance of the pathway correlations.

135

136

\subsection{Quantitative reverse transcription-polymerase chain reaction (RT-PCR) analysis}

RT-PCR was used to confirm the lncRNA expression profile data obtained from the microarray. Total RNA was isolated using the Trizol reagent (Life Technologies). Single-stranded cDNA was prepared from $2 \mu \mathrm{g}$ of total RNA, according to the manufacturer's instructions (Promega, USA), and the lncRNA expression was measured using quantitative PCR using SYBR Premix ExTaq. Two microliters of each cDNA was subjected to PCR amplification using primers specific for CUST_2117_PI428311958 (uc008nvu.1), CUST_4640_PI428311958 (AY621350), CUST_9613_PI428311958 (XR_009220.3), CUST_5105_PI428311958 (FQ225056), and CUST_6638_PI428311958 (BC058491). The lncRNA primers used in this study are shown in Table 1.

\subsection{LncRNA target prediction and IncRNA-mRNA co-expression network}

Differentially expressed lncRNAs were selected for target prediction, as previously described [17]. We used two independent algorithms to identify the target genes. The first algorithm searched for those acting in cis. The University of California Santa Cruz (UCSC) gene annotations (http://genome.ucsc.edu/) were used to pair and visualize the lncRNAs in the UCSC genome browser. All genes transcribed within a 10-kbp window up- or downstream of the lncRNA were considered cis-target genes. The second algorithm was based on mRNA sequence complementarity and RNA duplex energy prediction; it evaluated the impact of lncRNA binding on complete mRNA molecules using the BLAST software for first-round screening. The RNAplex software was used to screen target genes in trans ${ }^{[18]}$, with the RNAplex parameter set as $\mathrm{e} \leqslant-30$. Because the majority of identified LncRNAs functions were not clear, we established an lncRNA-mRNA co-expression network that comprised differentially expressed lncRNAs for cis- and trans-targeted mRNAs from the re-annotated Affymetrix Rats Genome Array data to reveal the connection between lncRNAs and mRNAs. 
159

160

161

162

163

164

165

166

167

168

169

170

171

172

173

174

175

176

177

178

179

180

181

182

183

184

185

\subsection{Statistical analysis}

Quantile normalization and subsequent processing of the raw data were performed using the GeneSpring Software GX 12.6.1 (Agilent technologies, Santa Clara, CA, US). The results were reported as mean \pm SEM from three independent tests. Student's $t$-test was performed using SPSS (13.0) to estimate the statistical significance of differences between groups. $\mathrm{P}<0.05$ was considered statistically significant.

3. Results:

\subsection{Effects of high-fat diet on glycolipid metabolism in SD rats}

We compared the fasting levels of blood glucose, low-density lipoprotein (LDL), high-density lipoprotein (HDL), and triglycerides (TG), as well as the body weight of DIO and normal rats. The results showed that the levels of HDL, LDL, and TG in the DIO group were not significantly higher than those in the control group. However, the fasting blood glucose level and body weight of the DIO rats were significantly higher than those of the control group (Figure 1, Supplementary Data S1).

\subsection{Effects of high-fat diet on sperm motility and testicular morphological structure in SD} rats

Semen analysis show that the sperm concentration and motility in the DIO group rats (53.63 \pm $13.82 * 10^{6} / \mathrm{ml}, 44.33 \pm 7.81 \%$, Respectively) was significantly lower than that in the normal control group $\left(92.18 \pm 6.99 * 10^{6} / \mathrm{ml}, 69.14 \pm 2.46 \%\right.$, Respectively) $(\mathrm{p}<0.05)$. In the normal control group, the testicular tissue showed that the sperm cells of the seminiferous tubules were normal and the sperm cells were arranged closely. The cell structure was clear at all stages, and a large number of mature spermatozoa were found in the lumen. At the same time, the testicular interstitial cells between seminiferous tubules can be clearly observed round and distributed in clusters; compared with the control group, the number of sperm cells of obese rats induced by high fat diet was decreased and arranged disorderly in the testes. The number of mature spermatozoa decreased significantly, and the sperm cells were found fallen in clusters in the lumen, sperms appeared deformity, and substantial cells were significantly reduced (Figure 2). 
186

187

188

189

190

191

192

193

194

195

196

197

198

199

200

201

202

203

204

205

206

207

208

209

210

211

212

\subsection{Expression levels of IncRNA and mRNA in the spermatozoa of DIO and normal rats}

Rat lncRNA Microarray (V6.0) is capable of detecting 23,260 lncRNAs and 26,623 mRNAs. In the present study, 9,843 lncRNAs and 23,183 mRNAs were detected in the sperm samples. After microarray scanning and normalization, 973 lncRNAs and 2,994 mRNAs were found to be differentially expressed, with fold change $\geqslant 2.0$ and $\mathrm{P}<0.05$. Among these, 457 and 516 lncRNAs were up-regulated and down-regulated, respectively, while, 1,316 and 1,678 mRNAs were up-regulated and down-regulated (fold change $\geqslant 2.0$ and $\mathrm{P}<0.05$ ), respectively, in the sperms of the DIO rats, compared with controls. Thirty-three lncRNAs displayed fold change $>$ 10, among which 16 were up-regulated and 17 were down-regulated (Supplementary Data S2; Table 2). CUST_6253_PI428311958 (fold change: 29.3) was the most up-regulated lncRNA, while CUST_3471_PI428311958 (fold change: 36.8) was the most down-regulated lncRNA in the sperms of DIO rats, compared with controls. Twenty-five mRNAs displayed fold change $>$ 15, among which 9 were up-regulated and 16 were down-regulated (Supplementary Data S3; Table 3). Visualization using scatter plots showed significant variations in the expression levels of lncRNAs and mRNAs (Figure 3).

\subsection{GO and pathway analysis}

LncRNAs are known to be involved in the function of the corresponding mRNA gene, and the mRNAs found to be significantly differentially expressed based on the GO enrichment analysis could reveal differences in the regulation of lncRNAs. In this study, prediction terms with pvalue $<0.01$ were selected and ranked based on the enrichment factor ([Count/ Pop. Hits]/[List. Total/Pop. Total]) or enrichment score (- $\log 10$ [p-value]). From our data, 263 BP terms, 39 CC terms, and $40 \mathrm{MF}$ terms were found $(\mathrm{p}<0.01)$ in the differentially expressed mRNAs (Supplementary Data S4). Here, we showed that the GO terms with the top 10 enrichment scores and those with the top 30 enrichment factors for differentially expressed mRNAs were associated with biological processes. Further, the cellular components were most relevant to an extracellular matrix component, the extracellular matrix. In addition, the GO terms for molecular function were correlated with growth factor binding, which is most important for sperm formation and 
213 semen quality (Figure 4A, B, C).

214 KEGG pathway analysis of mRNAs that were significantly differentially expressed was 215 performed to detect the pathways and molecular interactions associated with these genes. A total 216 of 38 important KEGG pathways were found with $\mathrm{P}$ value $<0.05$, and they were ranked based on 217 their enrichment scores (- $\log 10$ [p-value]) (Supplementary Data S5). Our data showed that the 218 pathways with the top 11 enrichment scores were associated with mRNAs. The metabolic 219 pathway was the top pathway in protein-coding genes such as "mucin type O-glycan 220 biosynthesis," "tyrosine metabolism," "protein digestion and absorption," "complement and 221 coagulation cascades," "drug metabolism-cytochrome P450," "peroxisome," and "carbon 222 metabolism." The result suggested that these pathways might contribute significantly to the 223 pathogenesis and development of DIO-associated male infertility (Figure 5).

$224 \quad 3.5$ Verification of the microarray data by RT-PCR

225 We randomly selected five dysregulated lncRNAs, including both up-regulated 226 (CUST_2117_PI428311958, CUST_4640_PI428311958, CUST_9613_PI428311958, 227 CUST_5105_PI428311958) and down-regulated (CUST_6638_PI428311958) ones, for verification with sperm samples from three other rats, using GAPDH and RPL19 as the internal standards. The dissolution curve analysis showed a single peak, indicating that the specificity of PCR amplification and sample triplet repeat was satisfactory. The results from the RT-PCR and microarray were consistent with each other. Thus, the results of qRT-PCR verified the accuracy of the microarray data, providing valid evidence that lncRNAs might play an important role in the pathogenesis of male infertility caused by DIO (Figure 6).

\subsection{Coding-non-coding gene network}

We established a form that included the differential expression lncRNAs for cis- (Supplementary Data S6) and trans- (Supplementary Data S7) targeted coding genes from the re-annotated Affymetrix Rat Genome Array data. In addition, we selected four up-regulated lncRNAs, CUST_2117_PI428311958 (uc008nvu.1), CUST_4640_PI428311958 (AY621350), CUST_5105_PI428311958 (FQ225056), and CUST_6805_PI428311958 (FQ212903), and two 
240

240

241

242

243

244

245

246

247

248

249

250

251

252

253

254

255

256

257

258

259

260

261

262

263

264

265

266

down-regulated lncRNAs,

CUST_1425_PI428311958

(uc008cdl.2)

and CUST_6637_PI428311958 (AF139830), for cis- and trans-targeted gene prediction. We then established an lncRNA-mRNA network. Through target gene prediction, target genes of the 26 aforementioned mRNAs were detected (Figure 7).

\section{Discussion:}

Infertility refers to the condition suffered by a couple who could not get pregnant despite 1 year of healthy sexual life without the use of contraceptive measures. The incidence of infertility has been significantly increasing, and male infertility accounts for $25-30 \%$ of it ${ }^{[19]}$. Studies have shown that male fertility may be severely affected by changes associated with obesity, type II diabetes, and metabolic syndrome ${ }^{[20-22]}$. Obesity and male infertility are known to be closely related, with the incidence of infertility in obese men being significantly higher than that in normal males [23]. The effect of obesity on male reproductive capacity is complex and multifaceted. Studies have shown that obesity can cause sexual retardation ${ }^{[24]}$, while increased body mass index (BMI) has been shown to have a negative impact on the levels of luteinizing hormone, testosterone, gonadotropin, sex hormone binding protein, and estradiol in men ${ }^{[25,26]}$. Some studies have also shown that obesity can cause erectile dysfunction, affecting the volume, concentration, activity, and count of sperms. Obesity is also closely associated with increased sperm DNA damage [27-29]. Thus, there is a considerable amount of evidence for a strong correlation between obesity and male infertility. Therefore, studying the effect of obesity on the mechanism and pathophysiological process of male infertility has high clinical value.

LncRNAs generally have no coding potential and are longer than $200 \mathrm{nt}$. Originally, lncRNAs were considered the "noise" of genome transcription, with no biological function [30]. However, many studies have recently demonstrated that lncRNAs play important roles in regulating gene expression by epigenetic, transcriptional, and post-transcriptional regulation; they have also been shown to affect cell proliferation, differentiation, metabolism, and apoptosis [31,32]. LncRNAs were found to be differentially expressed in sperm samples from obese and normal subjects, indicating that it might be a target for therapy against obesity-associated male 
267 infertility. In this study, we confirmed that lncRNAs were differentially expressed in sperm from 268 DIO and normal rats. We also explored the effect of obesity on reproduction at the molecular 269 level and the effect of lncRNAs on obesity and male reproduction.

270 In this study, we performed a comprehensive analysis of dysregulated lncRNAs by 271 comparing the transcriptome profiles of sperm samples from obese and normal rats. A total of 272973 lncRNAs were discovered. Among these, 457 were up-regulated and 516 were down273 regulated; we extracted their general features. We selected three up-regulated 274 (CUST_2117_PI428311958, CUST_9613_PI428311958, and CUST_5105_PI428311958) and 275 two down-regulated (CUST_6638_PI428311958, CUST_396_PI428311958) lncRNAs for 276 verification using qRT-PCR. The results of the qRT-PCR analysis were consistent with the 277 microarray results, indicating that the microarray data was reliable. Thus, our study provided a comprehensive understanding of the role of lncRNAs in DIO-induced male infertility; our results could help understand the epigenetic effects of lncRNAs on male infertility in obese patients.

GO term enrichment analysis was performed to identify biological processes, cellular components, and molecular functions associated with the differentially expressed lncRNAs. We found that the differentially expressed lncRNAs were highly enriched in functions related to biological process such as negative regulation of reproductive processes and regulation of endothelial cell proliferation; cell components such as extracellular matrix component, basement membrane, and scavenger receptor activity; and molecular functions such as growth factor binding. All these were closely associated with male infertility. In addition, pathway analysis showed a significant change in metabolic pathways, mucin type-O-Glycan biosynthesis, protein digestion and absorption, tyrosine metabolism, glycosphingolipid biosynthesis-ganglio series, and cytokine-cytokine receptor interaction. These results suggested that metabolic, endocrine, and other abnormalities might affect obese patients.

In this study, we found many dysregulated lncRNAs in the sperm samples of rats with DIO, and predicted their corresponding mRNAs through cis- and trans-targeting. For example, among the detected mRNAs in cis, up-regulated lncRNA CUST_2117_PI428311958 (uc008nvu.1; fold 
294

295

296

297

298

299

300

301

302

303

304

305

306

307

308

309

310

311

312

313

314

315

316

317

318

319

320

change: 8.12) was predicted to act on Wfdc3 (Supplementary Data Table S6), which is a WFDC type serine protease inhibitor located on human chromosome 20. Studies have shown that Wfdc3 is highly expressed in the epididymis, sperm, testes, and other male reproductive organs. It therefore plays a potential role in male fertility ${ }^{[33]}$. Wfdc3 was the predicted target gene for hsamiR-487a, which has been detected in the microRNA expression profile of the sperm of patients with asthenospermia ${ }^{[34]}$. However, the role of Wfdc3 in the reproductive process has not been extensively studied, and the association of mutations in the WFDC protease inhibitor gene with male infertility need to further studied. Furthermore, the microarray analysis had predicted that CUST_5105_PI428311958 (FQ225056; fold change: 5.87) would act both in cis (Ccnd2) and trans (Ccnd2) (Supplementary Data Table S6, S7). Cend2 is associated with cellular regulation, and its dysregulated expression could lead to abnormal cell proliferation ${ }^{[35]}$. A previous study had confirmed that the risk of type 2 diabetes was halved by the presence of a low-frequency allele in lncRNA-CCND2 that promoted insulin secretion ${ }^{[36]}$. Thus, CUST_2117_PI428311958 and CUST_5105_PI428311958 could be mediators for the occurrence and progression of obesity-associated male infertility. However, due to the lack of known function of lncRNAs, lncRNA-mRNA interactions should be studied in detail. It would be particularly important to improve our understanding of the mechanisms behind lncRNA-associated diseases and available techniques to diagnose and prevent them.

In this study, we constructed and analyzed the expression patterns of mRNAs and lncRNAs in DIO and normal rats. Our results revealed many important lncRNAs, whose expression levels affected the development of obesity. Further studies will be necessary to investigate the molecular mechanisms of action of specific lncRNAs, which could help in the exploration of novel therapeutic targets in DIO-associated male infertility.

\section{Conclusions:}

In summary, we detected the abnormal expression of lncRNAs and mRNAs in the sperm samples of DIO rats, and analyzed the potential roles of mRNAs through bioinformatics. The GO term enrichment analysis showed that the function most highly enriched was related to negative 
321 regulation of reproductive processes. Pathway analysis showed that metabolic pathways might be 322 related to the obesity-induced decline in male fertility. Our results would be helpful for future

323 studies that investigate the molecular role of lncRNAs in DIO-associated male infertility. We

324 provided experimental data on male infertility caused by obesity, and the lncRNA expression 325 profile that we constructed could contribute to future studies that investigate the molecular 326 functions of lncRNAs in obesity-associated decrease in male fertility.

327 6. Acknowledgements:

328 The authors would like to acknowledge Bo Hao, Shanghai, China, were the microarray 329 experiments were performed.

330 7. References:

331[1] Adams J P, Murphy P G. Obesity in anaesthesia and intensive care. [J]. BJA: British Journal 332 of Anaesthesia, 2000, 85(1):91-108.

333[2] Kushner R F, Bessesen D H. Treatment of the Obese Patient. [M]. Humana Press, 2007.

334[3] Swan S H, Elkin E P, Fenster L. The Question of Declining Sperm Density Revisited: An 335 Analysis of 101 Studies Published 1934-1996[J]. Environmental Health Perspectives, 2000, 336 108(10):961-6.

337[4] Landry D, Cloutier F, Martin L J. Implications of leptin in neuroendocrine regulation of 338 male reproduction. [J]. Reproductive biology, 2013, 13(1):1-14.

339[5] Yan W, Mu Y, Yu N, et al. Protective effects of metformin on reproductive function in 340 obese male rats induced by high-fat diet.[J]. Journal of Assisted Reproduction and Genetics, $3412015,32(7): 1-8$

342[6] Ferramosca A, Conte A, Moscatelli N, et al. A high-fat diet negatively affects rat sperm 343 mitochondrial respiration. [J]. Andrology, 2016, 4(3):520.

344[7] Cui X, Long C, Tian J, et al. Protective Effects of Fluvastatin on Reproductive Function in 345 Obese Male Rats Induced by High-Fat Diet through Enhanced Signaling of mTOR. [J]. 346 Cellular Physiology \& Biochemistry International Journal of Experimental Cellular 347 Physiology Biochemistry \& Pharmacology, 2017, 41(2):598.

348[8] Kriegel T M, Heidenreich F, Kettner K, et al. Identification of diabetes- and obesity349 associated proteomic changes in human spermatozoa by difference gel electrophoresis.[J]. 350 Reproductive Biomedicine Online, 2009, 19(5):660-670. 
351[9] Cabler S, Agarwal A, Flint M, et al. Obesity: modern man's fertility nemesis. [J]. Asian 352 Journal of Andrology, 2010, 12(4):480-9.

35[10] Gibb E A, Brown C J, Wan L L. The functional role of long non-coding RNA in human 354 carcinomas. [J]. Molecular Cancer, 2011, 10(1):38-38.

35[1 1] Ma L, Bajic V B, Zhang Z. On the classification of long non-coding RNAs. [J]. Rna Biology, 356 2013, 10(6):925-33.

35[12] Akinobu M, Alessandra P, Masaki M, et al.TORC1 and muscle regeneration are regulated by the LINC00961-encoded SPAR polypeptide. [J]. Nature, 2016, doi:10.1038/nature21034

35[13] Wu H J, Zhang C Y, Zhang S, et al. Microarray Expression Profile of Circular RNAs in Heart Tissue of Mice with Myocardial Infarction-Induced Heart Failure.[J]. Cellular Physiology \& Biochemistry International Journal of Experimental Cellular Physiology

36814] Rinn J L, Chang H Y. Genome regulation by long noncoding RNAs. [J]. Biochemistry, 2012, 364 81(81):145-166.

36[15] Bao J, Wu J, Schuster A S, et al. Expression Profiling Reveals Developmentally Regulated lncRNA Repertoire in the Mouse Male Germline[J]. Biology of Reproduction, 2013,

36[16] Jiang G J, Zhang T, An T, et al. Differential Expression of Long Noncoding RNAs between 89(5):000062.

37818] Tafer H, Hofacker I L. RNAplex: a fast tool for RNA-RNA interaction search[C]// 2008; 2657-2663.

37[19] Jensen T K, Andersson A M, Jørgensen N, et al. Body mass index in relation to semen quality and reproductive hormones among 1,558 Danish men.[J]. Human Reproduction,

37[20] Hammoud A O, Gibson M, Peterson C M, et al. Obesity and Male Reproductive Potential. [J]. Journal of Andrology, 2006, 27(5):619-26.

38[21] Pasquali R. Obesity and androgens: facts and perspectives. [J]. Fertility \& Sterility, 2006, 85(85):1319-1340. 
38522] Ghanbari E, Nejati V, Najafi G, et al. Study on the Effect of Royal Jelly on Reproductive 383 Parameters in Streptozotocin-Induced Diabetic Rats. [J]. International Journal of Fertility \& $384 \quad$ Sterility, 2015, 9(1):113-20.yyh

38[23] Sermondade N, Faure C, Fezeu L, et al. BMI in relation to sperm count: an updated 386 387 systematic review and collaborative meta-analysis. [J]. Human Reproduction Update, 2013, 19(3):221-231.

Lee J M, Kaciroti N, Appugliese M D, et al. Body Mass Index and Timing of Pubertal Initiation in Boys. [J]. Jama Pediatrics, 2010, 164(164):139-44. men.[J]. Human Reproduction, 2015, 30(12):2713-24. Fui M N, Dupuis P, Grossmann M. Lowered testosterone in male obesity: mechanisms, morbidity and management. [J]. Asian Journal of Andrology, 2014, 16(2):223-231.

Pan L, Ma J, Pan F, et al. Long Non-Coding RNA Expression Profiling in Aging Rats with Erectile Dysfunction. [J]. Cellular Physiology \& Biochemistry, 2015, 37(4):1513-1526. Magnusdottir E V, Thorsteinsson T, Thorsteinsdottir S, et al. Persistent organochlorines, sedentary occupation, obesity and human male subfertility.[J]. Human Reproduction, 2005, 20(1):208-215.

40ß331] Caley D P, Pink R C, Trujillano D, et al. Long noncoding RNAs, chromatin, and development.[J]. The scientific world journal, 2010, 10(1):90-102. Molecular Medicine, 2014, 92(4):337-346. Jalkanen J, Kotimaki M I, Poutanen M. Novel epididymal protease inhibitors with Kazal or WAP family domain. [J]. Biochemical \& Biophysical Research Communications, 2006,

41[34] Landgraf $P$, Rusu M, Sheridan R, et al. A mammalian microRNA expression atlas based on 411 small RNA library sequencing. [J]. Cell, 2007, 129(7):1401-1414.

41[35] Dong Q, Meng P, Wang T, et al. MicroRNA let-7a inhibits proliferation of human prostate 

cancer cells in vitro and in vivo by targeting E2F2 and CCND2.[J]. Plos One, 2010, 5(4):e10147.

41536] H Y, A S, Rm F, et al. Association analysis of 29,956 individuals confirms that a low 416 frequency variant at CCND2 halves the risk of type 2 diabetes by enhancing insulin 417 secretion. [J]. Diabetes, 2015, 64(6):2279-85.

8. Figure legends

421

Figure 1. Effect of high-fat diet-induced obesity (DIO) on the levels of fasting blood glucose (GLU), HDL, LDL, and TG, as well as body weight. Data are expressed as mean \pm SEM. **p $<0.01, * \mathrm{p}<0.05$, compared with the control group, $\mathrm{n}=7$.

425

Figure 2. HE staining in the testis of control and high fat diet fed SD rat. (original

Figure 3. Scatter plots assessing the variation in expression of IncRNAs (A) and mRNAs (B) in the two compared groups. $\mathrm{X}$ - and $\mathrm{Y}$-axes represent averaged normalized signal values of the microarray samples of the control and experimental groups. LncRNAs and mRNAs below the blue line and above the red line showed greater than 2.0-fold variation in expression between the two groups.

Figure 4. GO analysis of differentially expressed mRNAs. Top 10 GO terms BP (A) and MF (B) ranked by enrichment scores are shown. (C) GO annotations of differentially expressed mRNAs with top 30 enrichment factors ([Count/Pop. Hits]/[List. Total/Pop. Total]) covering domains of biological processes (BP, circles), molecular functions (MF, squares), and cellular components (CC, triangles). Size represents the number of enriched genes and color indicates the 440 degree of enrichment. 
442 Figure 5. KEGG pathway analysis of differentially expressed mRNAs. Analysis of the 443 enrichment scores ( $-\log 10$ [P value]) of differentially expressed mRNAs with top 11 terms.

444

Figure 6. Validation of microarray data by qRT-PCR. Comparison of the results of qRT-PCR 446 and microarray for lncRNAs. Results obtained with these two methods were consistent with each 447 other. (A) Gapdh; (B) RPL19; (C) Array.

448

449

Figure 7. LncRNA-mRNA network. Blue square nodes and pink round nodes represent 450 mRNAs and lncRNAs, respectively; purple dashed lines and blue solid lines between two nodes represent trans- and cis-targets, respectively. Size of the points indicates the number of targets 452 associated with the lncRNAs. 
Figure 1

Effect of high fat diet induced obesity (DIO) on GLU, HDL, LDL and TG. Data are expressed as mean \pm SEM. $* * p<0.01$ compared with the control group, $\mathrm{n}=7$.

A

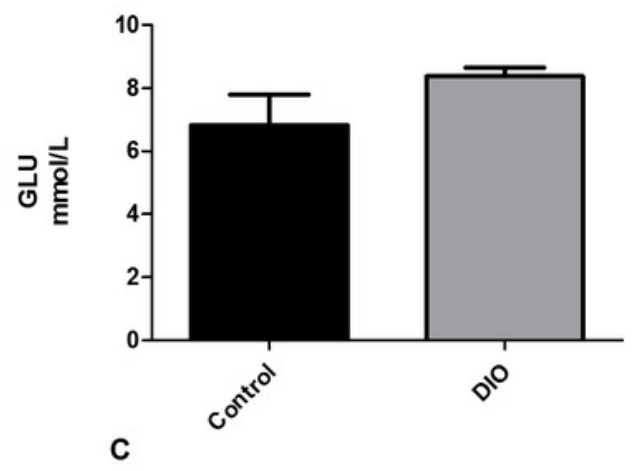

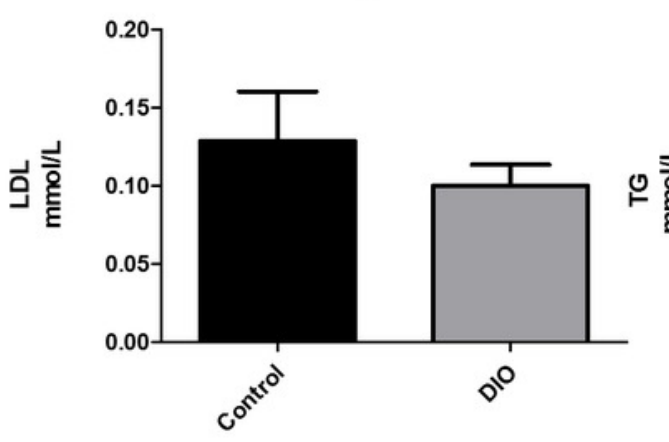

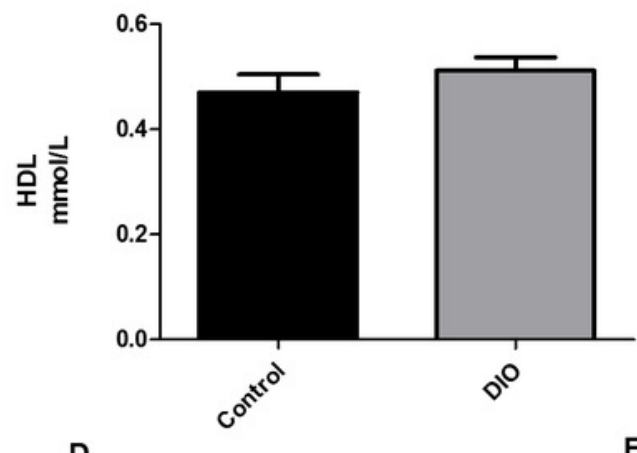

D
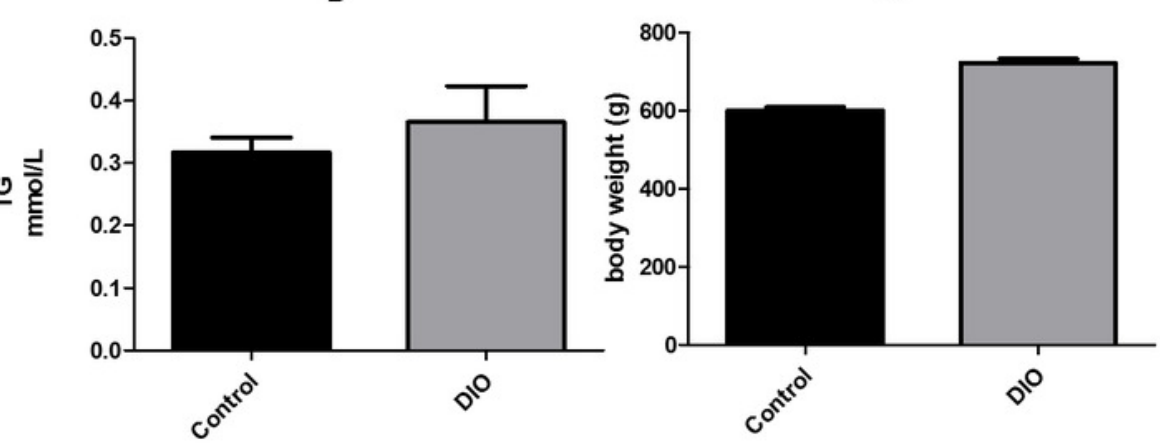
Figure 2

HE staining in the testis of control and high fat diet fed SD rat (original magnification, 20x and 40x).

\section{Control}
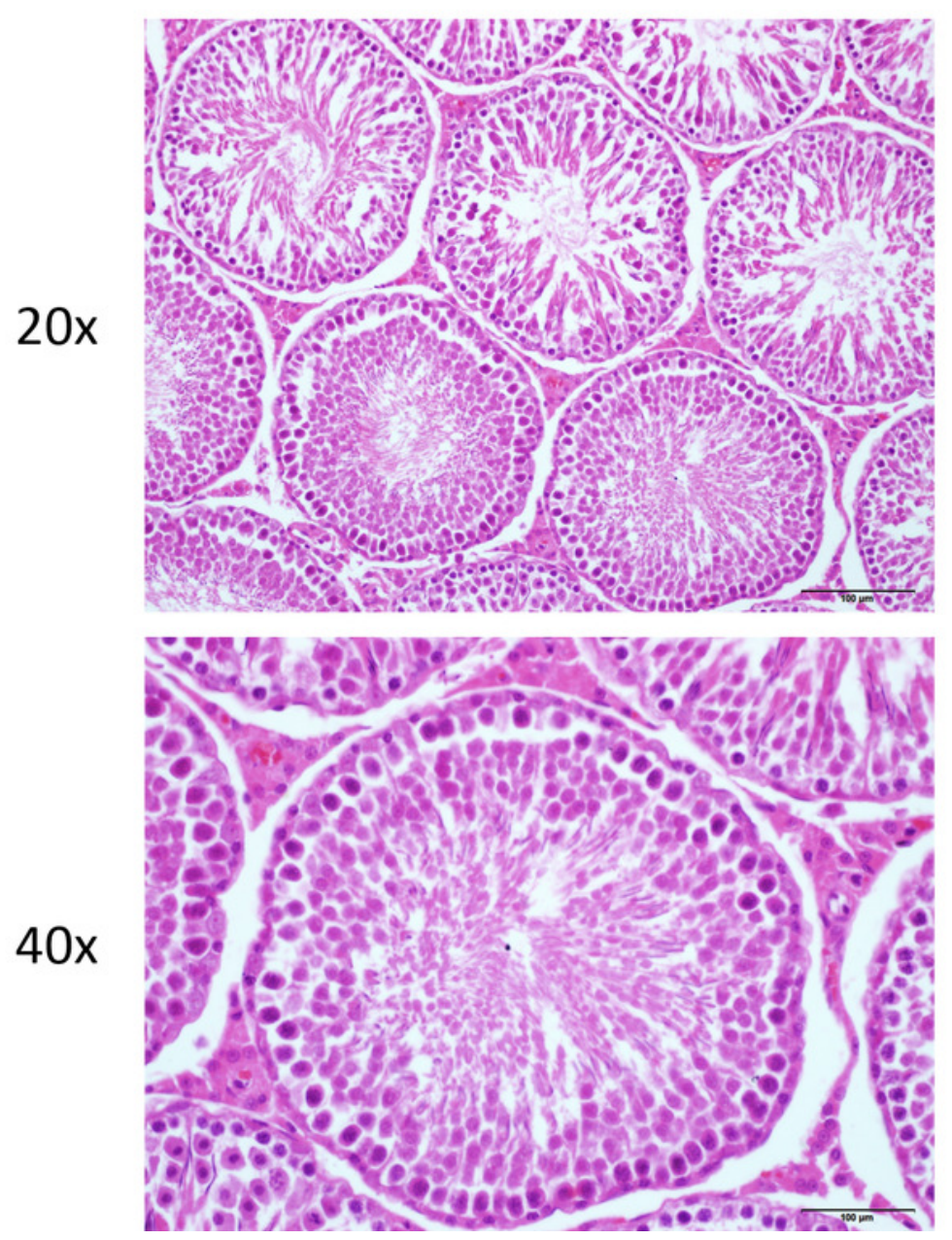

DIO
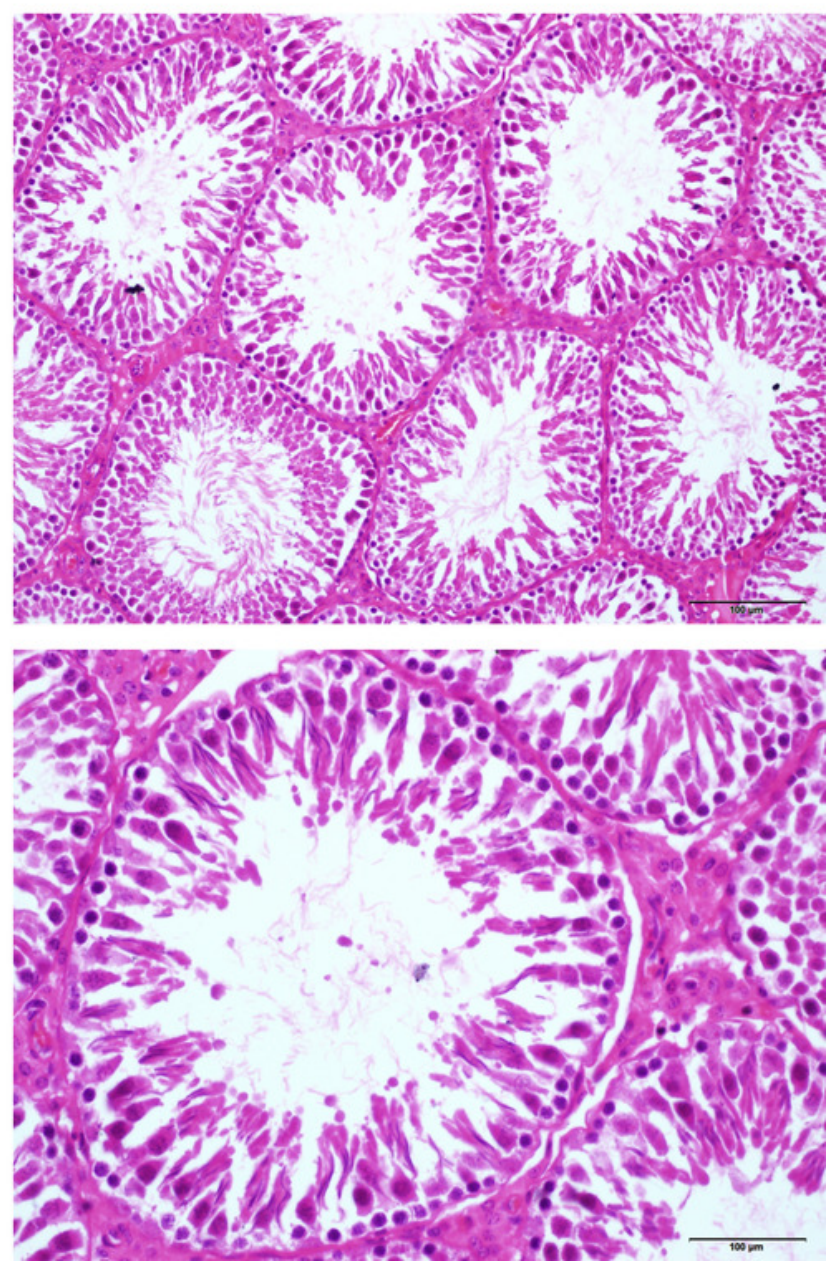


\section{Figure 3}

Scatter plots assessing the variation in expression of IncRNAs (A) and mRNAs (B) in the two compared groups.

$\mathrm{X}$ - and $\mathrm{Y}$-axes represent averaged normalized signal values of the microarray samples of the control and experimental groups. LncRNAs and mRNAs below the blue line and above the red line showed greater than 2.0-fold variation in expression between the two groups.
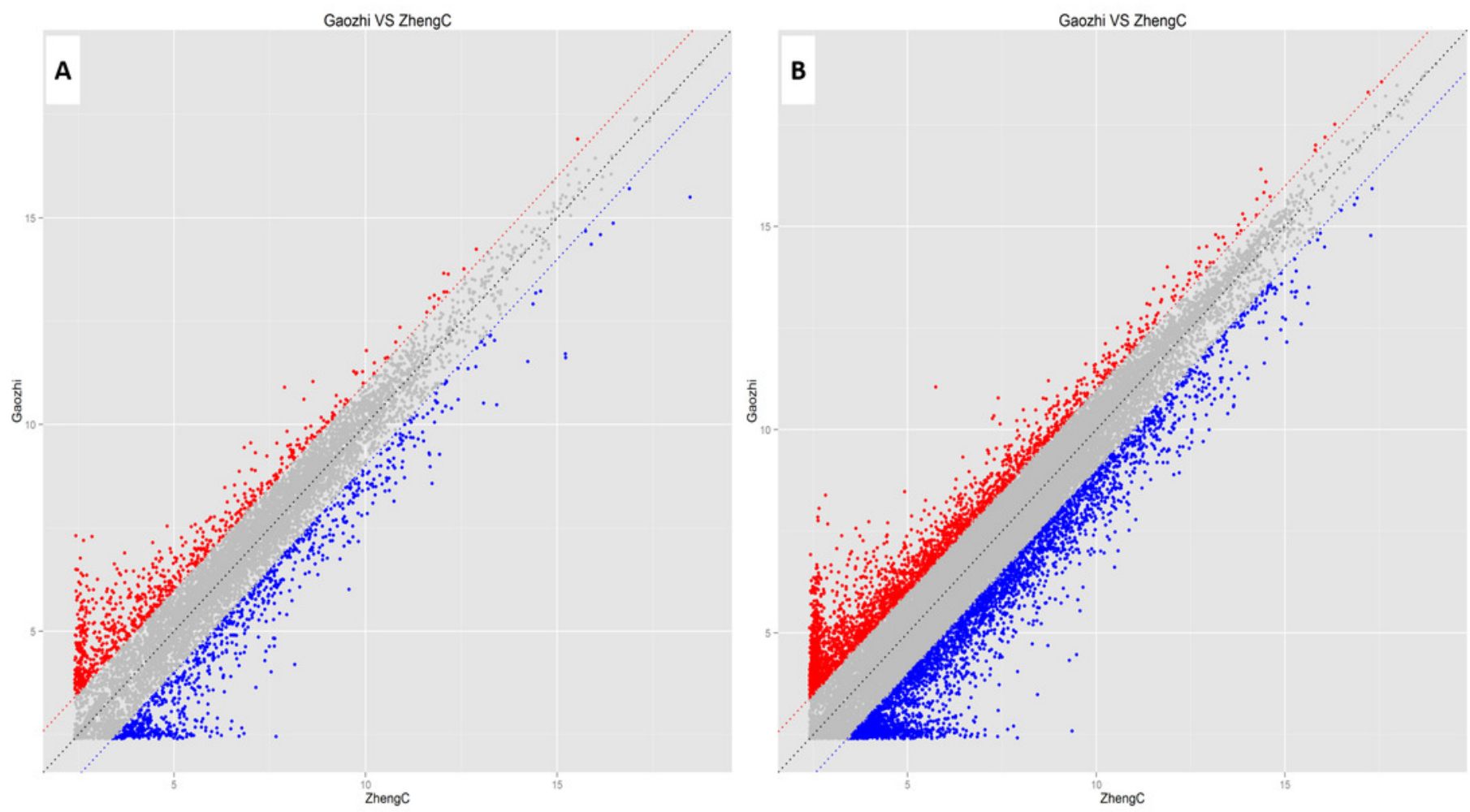


\section{Figure 4}

GO analysis of differentially expressed mRNAs.

Top $10 \mathrm{GO}$ terms BP (A) and MF (B) ranked by enrichment scores are shown. (C) GO annotations of differentially expressed mRNAs with top 30 enrichment factors ([Count/Pop. Hits]/[List. Total/Pop. Total]) covering domains of biological processes (BP, circles), molecular functions (MF, squares), and cellular components (CC, triangles). Size represents the number of enriched genes and color indicates the degree of enrichment. 
A

single-organism process

developmental process

single-organism developmental process

single-organism cellular process

cellular process

multicellular organismal development

anatomical structure development

system development

localization

organ development

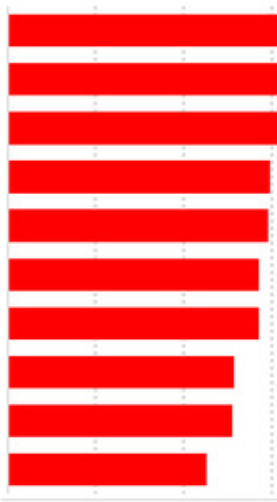

10

2030

40

Biological Process Enrichment Score(-log10(Pvalue))
B

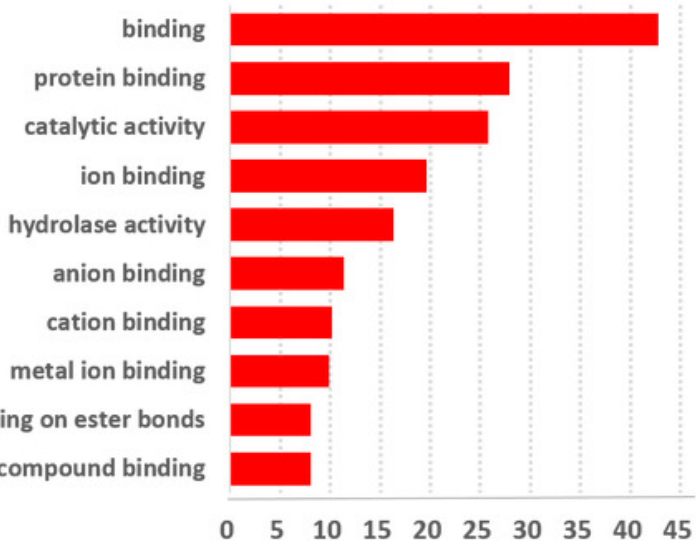

Molecular Function Enrichment Score(-log10(Pvalue))

sulfur compound binding

$\begin{array}{llllllllll}0 & 5 & 10 & 15 & 20 & 25 & 30 & 35 & 40 & 45\end{array}$

C

\begin{abstract}
sulfur compound binding
stem cell development

spermatid differentiation -

scavenger receptor activity -

regulation of wound healing -
\end{abstract}

regulation of leukocyte migration -

regulation of endothelial cell proliferation -

regulation of chemotaxis -

proteinaceous extracellular matrix -

positive regulation of leukocyte migration -

positive regulation of chemotaxis -

positive regulation of behavior -

negative regulation of reproductive process -

negative regulation of cell achesion -

negative regulation of cell activation -

myeloid leukocyte migration mesenchymal cell differentiation mesenchymal coll development -

leukocyte migration leukocyle chemotaxis -

heparin binding -

hemostasis -

growth factor binding *

glycosaminoglycan binding -

extracellular matrix component -

extracellular matrix -

digestive tract development -

defense response to bacterium -

cell chemotaxis -

basement membrane -
Top 30 of GO Enrichment

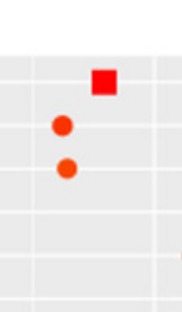

○

$\Delta$

.

-

-

hydrolase activity, acting on ester bonds
50 


\section{Figure 5}

KEGG pathway analysis of differentially expressed mRNAs.

Analysis of the enrichment scores (-log 10 [P value]) of differentially expressed mRNAs with top 11 terms.

\section{Enrichment score (- $\log (\mathbf{P}$ Value $))$}

1

2

3

4

5

6

7

8

Metabolic pathways

Mucin type O-Glycan biosynthesis

Protein digestion and absorption

Tyrosine metabolism

Glycosphingolipid biosynthesis - ganglio series

Complement and coagulation cascades

Peroxisome

Carbon metabolism

Drug metabolism - cytochrome P450

Glycine, serine and threonine metabolism

Glycolysis / Gluconeogenesis
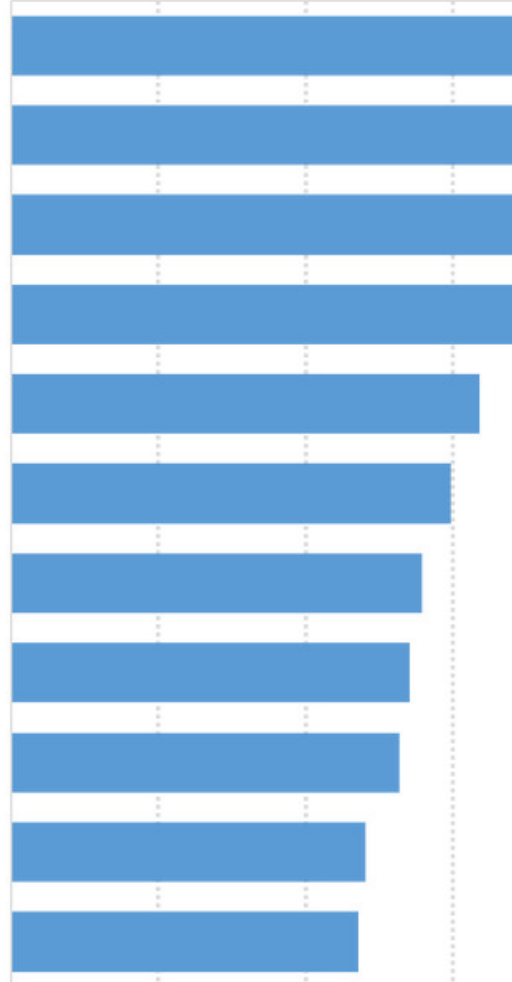


\section{Figure 6}

Validation of microarray data by qRT-PCR.

Comparison of the results of qRT-PCR and microarray for IncRNAs. Results obtained with these two methods were consistent with each other. (A) Gapdh; (B) RPL19; (C) Array. 

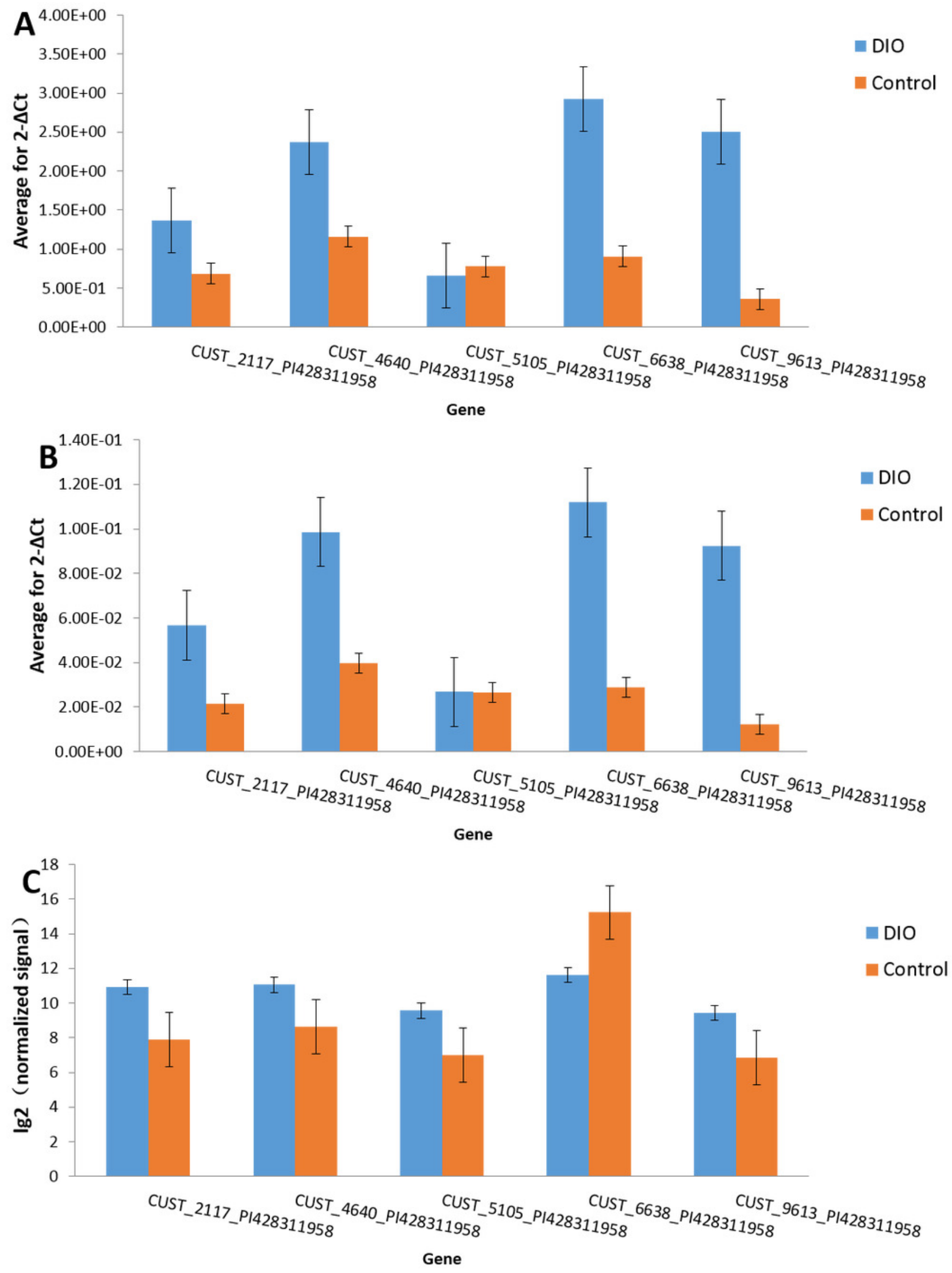


\section{Figure 7}

LncRNA-mRNA network.

Blue square nodes and pink round nodes represent mRNAs and IncRNAs, respectively; purple dashed lines and blue solid lines between two nodes represent trans- and cis-targets, respectively. Size of the points indicates the number of targets associated with the IncRNAs.
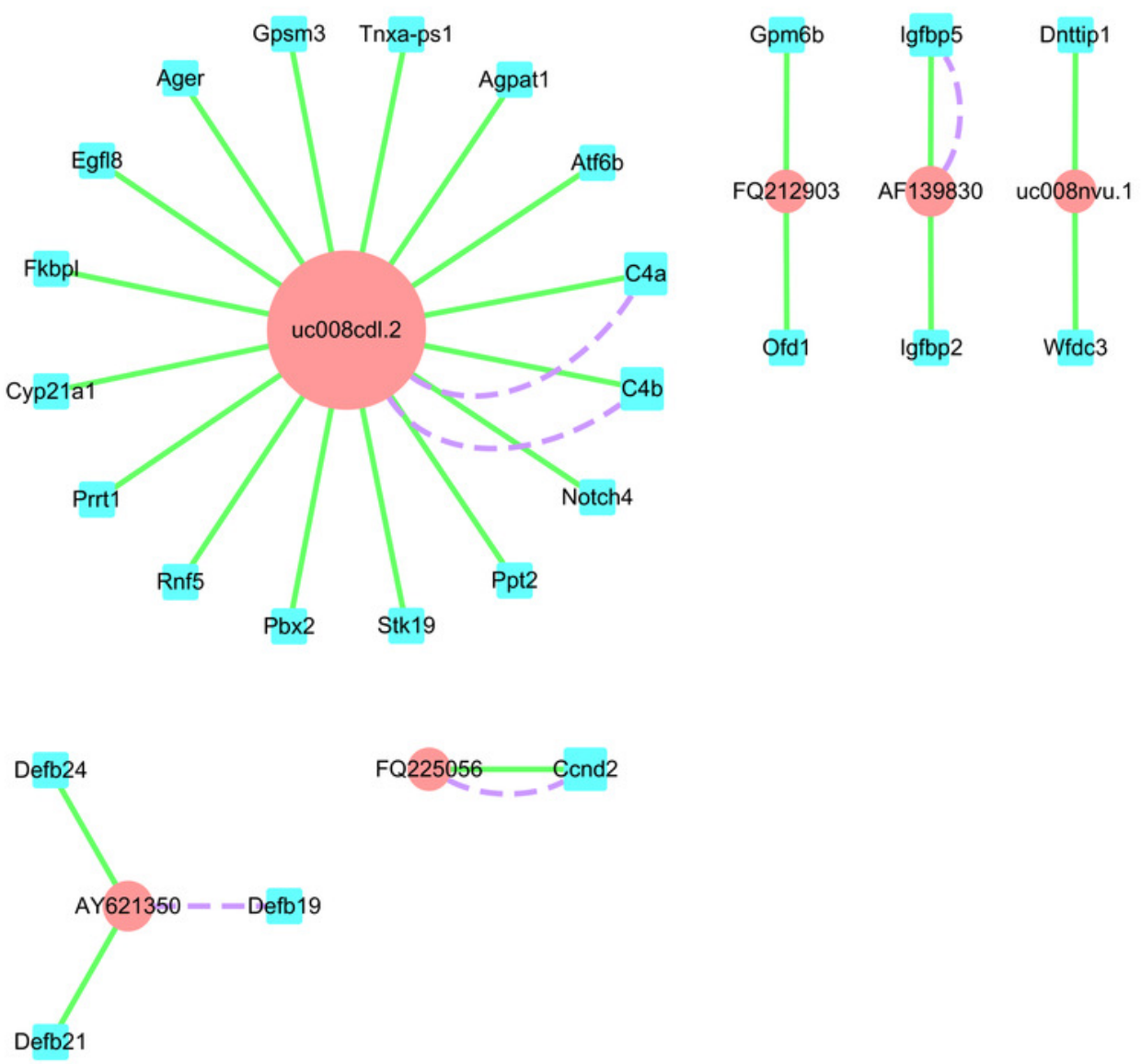
Table $\mathbf{1}$ (on next page)

Specific IncRNA primers for quantitative PCR analysis. 
1 Table 1. Specific lncRNA primers for quantitative PCR analysis

\begin{tabular}{ll}
\hline Primer Name & Sequence(5'to3') \\
\hline CUST_2117_PI428311958-F & ATCCTGGGGTTTGTGACACT \\
CUST_2117_PI428311958-R & GGAAAGAGAAGCACCCATCA \\
CUST_4640_PI428311958-F & AGCAACGGGGACTACTGCT \\
CUST_4640_PI428311958-R & GTTCTTGAGGACCGCCACT \\
CUST_5105_PI428311958-F & GCAGGTGATTGGCTCCTAAGTC \\
CUST_5105_PI428311958-R & CAGATAACAGTGGGAAACGTCTACA \\
CUST_6638_PI428311958-F & CACCCTTCTCCGGACTTCCT \\
CUST_6638_PI428311958-R & GGACCCCAACACCTCTTTTCT \\
CUST_9613_PI428311958-F & CACACAAGCATCCCCACAG \\
CUST_9613_PI428311958-R & ATTGCGTGTGTATGTCTTTCCA \\
Rpl19(RAT15138)-F & TCCAAGGAGGAAGAGACCAA \\
Rpl19(RAT15138)-R & ACAAGGACGAAGGCTTGTTT \\
Gapdh-F & GGCCTCTCTCTTGCTCTCAGTATC \\
Gapdh-R & \\
\hline
\end{tabular}


Table 2 (on next page)

Differentially expressed IncRNAs (Foldchange $>15, \mathrm{P} \leq 0.05$ ). 
1 Table 2 Differentially expressed $\operatorname{lncRNAs}$ (Foldchange $>15, \mathrm{P} \leq 0.05$ ).

\begin{tabular}{cccccc}
\hline ProbeName & foldchange & Regulation & ProbeName & foldchange & Regulation \\
\hline CUST_6253_PI428311958 & 29.30 & up & CUST_3471_PI428311958 & 36.85 & down \\
CUST_8359_PI428311958 & 23.57 & up & CUST_458_PI428311958 & 19.62 & down \\
CUST_8135_PI428311958 & 21.67 & up & CUST_5519_PI428311958 & 18.85 & down \\
CUST_787_PI428311958 & 18.58 & up & CUST_2111_PI428311958 & 17.15 & down \\
CUST_5947_PI428311958 & 16.69 & up & CUST_9135_PI428311958 & 15.42 & down \\
CUST_446_PI428311958 & 16.08 & up & CUST_8347_PI428311958 & 14.33 & down \\
CUST_5041_PI428311958 & 14.26 & up & CUST_2931_PI428311958 & 13.49 & down \\
CUST_305_PI428311958 & 13.14 & up & CUST_4808_PI428311958 & 12.80 & down \\
CUST_6233_PI428311958 & 12.77 & up & CUST_6638_PI428311958 & 12.14 & down \\
CUST_7638_PI428311958 & 12.44 & up & CUST_4899_PI428311958 & 12.02 & down \\
CUST_279_PI428311958 & 11.95 & up & CUST_396_PI428311958 & 11.75 & down \\
CUST_3553_PI428311958 & 11.51 & up & CUST_8936_PI428311958 & 11.45 & down \\
CUST_3255_PI428311958 & 10.33 & up & CUST_6637_PI428311958 & 11.32 & down \\
CUST_7370_PI428311958 & 10.24 & up & CUST_8558_PI428311958 & 11.27 & down \\
CUST_2918_PI428311958 & 10.23 & up & CUST_6993_PI428311958 & 11.18 & down \\
CUST_677_PI428311958 & 10.17 & up & CUST_4393_PI428311958 & 10.99 & down \\
& & & CUST_8371_PI428311958 & 10.60 & down \\
\hline
\end{tabular}


Table 3 (on next page)

Differentially expressed mRNAs (Foldchange $>15, \mathrm{P} \leq 0.05$ ). 
1 Table 3 Differentially expressed mRNAs (Foldchange $>15, \mathrm{P} \leq 0.05$ ).

\begin{tabular}{llll}
\hline ProbeName & foldchange & Regulation & GeneSymbol \\
\hline RAT03432 & 47.38 & up & Pga5 \\
RAT02407 & 42.69 & up & Gabrg1 \\
RAT07570 & 39.46 & up & Spink3 \\
RAT02312 & 35.21 & up & Hao2 \\
RAT05103 & 27.80 & up & Cc121 \\
RAT08360 & 22.52 & up & RGD1563982 \\
RAT25028 & 16.13 & up & B3galt6 \\
RAT15628 & 15.98 & up & Hgd \\
RAT10614 & 15.55 & up & Mrpl35 \\
RAT08939 & 108.82 & down & Olr705 \\
RAT05594 & 44.67 & down & Kcna2 \\
RAT27248 & 34.14 & down & Sertm1 \\
RAT05047 & 32.43 & down & Lrrn2 \\
RAT05303 & 32.12 & down & Sbsn \\
RAT03054 & 30.99 & down & RGD1560244 \\
RAT20303 & 30.99 & down & Olr440 \\
RAT28310 & 23.55 & down & Ptger3 \\
RAT04407 & 21.45 & down & Zp2 \\
RAT08234 & 20.19 & down & Olr96 \\
RAT21480 & 19.74 & down & Tnfrsf26 \\
RAT09493 & 18.27 & down & Itga7 \\
RAT18225 & 16.53 & down & Slc16a5 \\
RAT29116 & 16.21 & down & Adam26b \\
RAT04279 & 15.53 & down & Mpeg1 \\
RAT07795 & 15.51 & down & Fetub \\
\hline
\end{tabular}

2 